\title{
New Opportunities for Structural Biology Research at SSRL
}

Aina Cohen representing the entire SSRL-SMB team

acohen@slac.stanford.edu

Stanford Synchrotron Radiation Lightsource, Structural Molecular Biology Group, SLAC National Accelerator Laboratory, Menlo Park, California, 94025, USA

The Structural Molecular Biology (SMB) program at the Stanford Synchrotron Radiation Lightsource (SSRL) provides an integrated suite of macromolecular crystallography (MC) and small angle X-ray scattering (SAXS) beam lines enabling studies on the most challenging problems in structural biology. SSRL 12-1, a new next-generation microfocus beam line is in commissioning. BL12-1 is outfitted with a broad bandpass capability to provide exceptional brightness, smaller microbeams and a high number of reflections when rastering on the fly or using crystal injectors. It is equipped with a high frame rate EIGER detector and a highspeed goniometer, enabling new approaches for data collection and phasing.

Similarities in instrumentation and software environments will form the foundation of a synergistic relationship between the SSRL BL12-1 and the new Macromolecular Femtosecond crystallography instrument (MFX) at LCLS, through a Gateway approach. The standard sample environment available at MFX is a highly automated goniometer setup for diffraction experiments, developed and supported by the SMB group. The experimental front-end is based on developments at SSRL and LCLS XPP to provide an efficient framework to carry out goniometer-based experiments using automated strategies tailored to handle a variety of sample requirements, crystal sizes and experimental goals. These developments coupled with improvements in data processing algorithms make it possible to derive high-resolution crystal structures using only 100 to 1000 still diffraction images. 